\title{
Accurate Measurement of Molar Absorptivities
}

\author{
Robert W. Burnett \\ Clinical Chemistry Laboratory, Hartford Hospital, Hartford, Connecticut 06115
}

(June 7, 1972)

\begin{abstract}
The key to accurate measurement of molar absorptivities is a thorough understanding of the sources of error which appear throughout the measurement procedure. Sources of determinant error will be listed with comments on estimating their magnitude and eliminating them where possible. Sources of random error will be discussed as well as the propagation of both random and determinant errors. There is discussion of the need for accurate values of molar absorptivities using examples from clinical chemistry. Finally, the proper use of accurate absorptivity values in the clinical chemistry laboratory will be considered. Here, emphasis is on the need for a quality assurance system which includes routine checks on such things as wavelength calibration and photometric accuracy of spectrophotometers, calibration of analytical balances, and quality of incoming reagents.
\end{abstract}

Key words: Molar absorptivity in clinical chemistry; random errors in molar absorptivity; systematic errors in molar absorptivity.

\section{Introduction}

Absorption spectrophotometry in the visible and ultraviolet is undoubtedly one of the most valuable tools available to the analytical chemist. In clinical chemistry, roughly 60 percent of all determinations performed utilize this technique. It is natural, therefore, that in the quest for more meaningful data, considerable attention is focused on accuracy in spectrophotometry, and specifically on accurate measurement of molar absorptivities. In this paper the important sources of error which affect molar absorptivity measurements are reviewed and the propagation of the individual errors is discussed. In addition, some of the rationale underlying the increasing interest in accuracy in spectrophotometry is discussed, with particular reference to clinical chemistry.

The key to accurate measurement of molar absorptivities is careful attention to the various error sources which are present in the measurement process. The problem discussed here is that of establishing a molar absorptivity uncertainty which is no greater than one part per thousand. With this in mind, we will be concerned with individual sources of error down to a level of one or two parts per ten thousand, recognizing that many errors contribute to the final uncertainty. This point is demonstrated more formally in the brief discussion of error propagation. Much of the material presented on errors is in the nature of a review; it is thought that this may be useful since discussions of the various errors are somewhat scattered in the literature, and the mathematical complexity of some of the original discussions may obscure their practical implications. Reference is made to earlier papers for many of the mathematical details.

Molar absorptivity, $\epsilon$, may be defined by Beer's law as

$$
\epsilon=\frac{A M}{b c}
$$

where $A$ is absorbance, $M$ is the molecular weight of the solute, $b$ is the effective path-length in centimeters and $c$ is the solution concentration in grams per liter. The units of $\epsilon$ are thus $1 \cdot \mathrm{mol}^{-1} \cdot \mathrm{cm}^{-1}$. Two restrictions apply to eq (1); first, the equation is valid only for monochromatic radiation, and second, the ratio of absorbance to concentration is constant only for ideal solutions. The former restriction will be commented upon later and the latter is not usually a problem because measurements are ordinarily made at concentrations below $10^{-3} \mathrm{~mol} \cdot 1^{-1}$ where deviation from ideal behavior may be ignored. A related problem sometimes encountered is commonly known as a chemical deviation from Beer's law, which occurs when the concentration of the absorbing species does not increase linearly with solute concentration. Examples of this behavior are well known and the effect will not be commented upon further, except to note that the acid chromium (VI) system, which has been recently studied by Burke, et al. [1 $]^{1}$, is a particularly important system of this type because of its common use as an absorbance standard. It is emphasized that adherence to Beer's law over a range of concentrations 
should never be assumed in the absence of supporting experimental data. In contrast to the chemical sources of error, the items discussed below may be classed as instrumental and procedural sources of error in the measurement process.

${ }^{1}$ Figures in brackets indicate the literature references at the end of this paper.

\section{Systematic Errors}

\section{A. Gravimetric and Volumetric Errors}

Although these error sources may seem rather obvious, they deserve mention both for completeness and because certain components are probably often ignored. Analytical balances must be calibrated using weights certified by the National Bureau of Standards. The solution temperature at which absorbance measurements are made must be known and the solvent density at this temperature should be used in calculating the solution concentration. Accurate temperature measurement is especially important when the solvent has a high coefficient of thermal expansion; for example, chloroform shows a volume expansion of 1.3 parts per thousand per ${ }^{\circ} \mathrm{C}$ near room temperature. In addition, it is interesting to note that the relatively low heat capacity of many organic solvents means that they are much more sensitive to heating effects than is water. Chloroform shows 27 times the volume expansion of water for an equal heat input.

Where gravimetric technique is employed in measuring both solute and solvent, it is necessary to take the apparent specific volume of the solute into account in calculating the concentration, the relationship being

$$
c=\frac{1000 W_{x} \rho}{W_{s}+W_{x} \rho \bar{v}}
$$

where $c$ is concentration in grams per liter, $W_{x}$ is weight of solute in grams, $W_{s}$ is weight of solvent in grams, $\rho$ is solvent density in $\mathrm{g} / \mathrm{cm}^{3}$ and $\bar{v}$ is solute apparent specific volume in $\mathrm{cm}^{3} / \mathrm{g}$. This is especially important for solutes of relatively high molecular weight and low molar absorptivity, in which case the correction due to $\bar{v}$ can approach one part per thousand.

Finally, it must be remembered that in any weighing process which involves displacement of air, a buoyancy correction must be applied. The magnitude of this correction depends on the difference in density between the weights used and the material being weighed. For water, the correction is about one part per thousand and it is only slightly smaller for most solids. Buoyancy corrections for single-pan balances have been discussed in detail by Burg and Veith [2] and by Lewis and Woolf [3].

\section{B. Path Length Errors}

To obtain highly accurate absorbance measurements, the cell path length must obviously be known with very high accuracy. Electromechanical devices known as electronic gauges have been used for this purpose. In expert hands and with careful calibration and repetitive measurements, these instruments are capable of establishing the path length of a cell to $\pm 0.0001 \mathrm{~cm}$, which for a standard one-cm cell is a relative accuracy of one part per ten thousand. In addition, the measurement may be made at any point on the cell window so that the parallelism of the windows is also readily checked. The magnitude of the error which may be introduced by accepting the nominal value for cellpath length is not commonly appreciated. At the National Bureau of Standards, in a series of measurements of fourteen cells with a one-cm nominal pathlength, thirteen deviated from the nominal value by greater than one part per thousand and five were in error by greater than 1 percent [4]. As will be seen, this may easily be the most significant error source in a molar absorptivity measurement. An accurate measurement of cell path length will not suffice to eliminate all path length errors, however. Several additional factors which can influence the effective path length are discussed below.

\section{Beam Alignment Errors}

A significant error due to nonparallel incident radiation appears whenever the most extreme angle which the light rays make with a line normal to the cell windows exceeds about $3^{\circ}$, as shown in figure la.

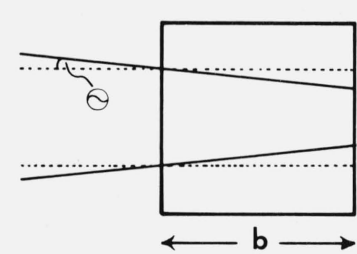

(a)

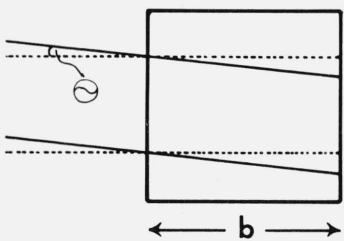

(b)
FigURE 1. (a) Increase in effective path length due to nonparallel incident radiation. (b) Increase in effective path length due to improper cell orientation.

This increases the effective path length and, therefore, the observed absorbance. At an absorbance of one, the error is five parts per thousand when the most extreme rays deviate from the normal by $10^{\circ}$; if the maximum deviation is $5^{\circ}$ the error is under one part per thousand. Exact corrections depend on the refractive index of the solution and must be obtained by numerical integration [5]. Proper cell orientation is also critical since cell windows must be normal to the incident radiation. Any other orientation will result in a longer light path through the cell, as shown in figure $1 \mathrm{~b}$. This effect is significant at deviations greater than $1^{\circ}$ and increases with $\Theta^{2}$, the approximate relation being

$$
\delta=\frac{\pi^{2} \Theta^{2}}{64,800 n^{2}}
$$


where $\delta$ is the fractional path length error, $\theta$ is the angle of incidence of the beam, in degrees, and $n$ is the refractive index of the solvent. The error function is shown graphically in figure 2 for $n=1.33$.

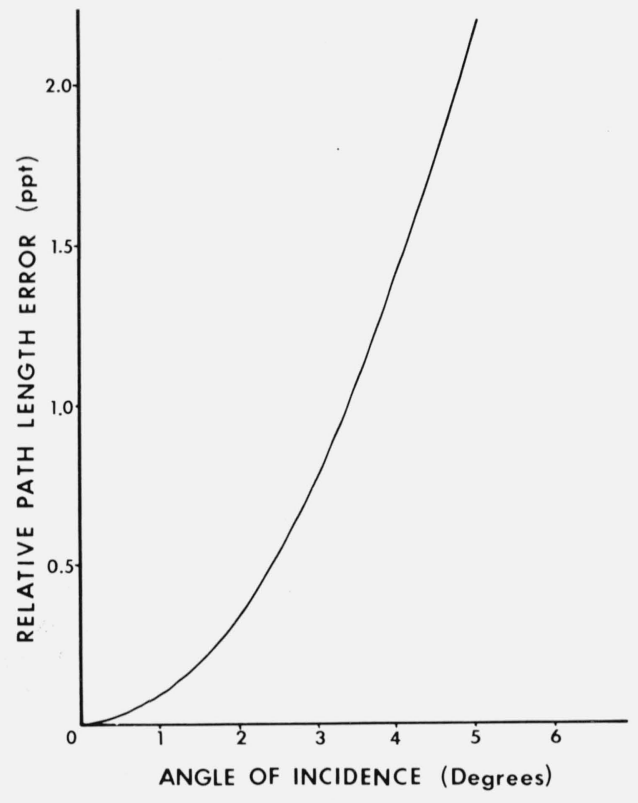

Figure 2. Relative path length error (parts per thousand) due to improper cell orientation, for $n=1.33$. From eq (3).

\section{Reflection Errors}

When light passes from one medium with refractive index $n_{1}$ to another with refractive index $n_{2}$, a fraction of the light is reflected at the interface. If the angle of incidence is zero (light beam normal to the interface) the Fresnel relation for the fraction reflected, is

$$
f=\left(\frac{n_{1}-n_{2}}{n_{1}+n_{2}}\right)^{2}
$$

An air-glass interface may be roughly represented by using $n_{\text {air }}=1.0$ and $n_{\text {glass }}=1.5$, in which case $f=0.04$, i.e. 4 percent of the incident light is reflected. Reflection losses can be partially compensated by subtracting the apparent absorbance of pure solvent from the absorbance of the solution in an identical cell. Unfortunately, this still leaves an important error which is due to multiple reflections within the cell. The major component of this error is due to light which enters the cell, is reflected back into the solution from the rear window (the window nearest the detector), is reflected again at the forward window, and reaches the detector having traversed the solution three times. This problem has been considered by Goldring,

et at. [6] who derived the following approximation for $\Delta A$, the increase in apparent absorbance due to multiple reflections,

$$
\Delta A=0.434\left(1-T^{2}\right)\left(\frac{f_{1} \cdot f_{2}}{1-f_{1}}\right)
$$

where $T$ is the solution transmittance, $f_{1}$ is the fraction of light reflected at the rear window (considering both interfaces) and $f_{2}$, the fraction reflected at the forward window. Equation (5) may be rewritten in terms of the relative absorbance error $\delta$,

$$
\delta=\frac{\left(0.434-e^{-2 A}\right)}{A}\left(\frac{f_{1} \cdot f_{2}}{1-f_{1}}\right)
$$

Figure 3 shows how $\delta$ varies with $A$ for the condition $f_{1}=f_{2}=0.04$. This should be recognized as the minimum error which is possible since only the reflections

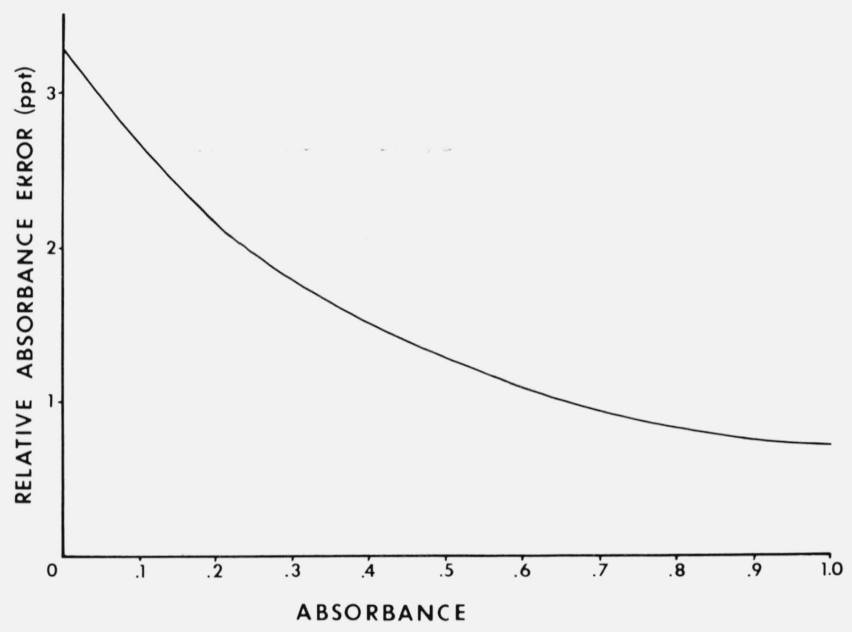

FigURE 3. Relative absorbance error (parts per thousand) due to multiple reflections at cell windows. From eq (6) with $f_{1}=f_{2}=0.04$.

at the air-glass interface are considered. In practice, it may be found that reflections from other surfaces close to the light path are significant, and $f_{1}$ and/or $f_{2}$ may be much greater than 0.04 [6], although this should not be the case in well-designed instruments.

\section{E. Effect of Finite Slit Width}

Since nomenclature in this subject is not completely standardized, the following definitions are given: slit function plot is the plot of photon flux emergent from the exit slit versus wavelength; spectral bandwidth is the width at half-height of the slit function plot; natural bandwidth is the width at half-height of the absorption band; bandwidth ratio is the ratio of the spectral bandwidth to the natural bandwidth. Since Beer's law is valid only for monochromatic light, the use of a band of radiation, albeit narrow, introduces an error unless the absorption spectrum is extremely flat in the region of interest. The mathematical formulation of this problem has been dealt with by several authors [7-9] and usually involves the assumptions of a triangular slit function plot and a Gaussian shape for the absorption band. The results obtained show that the observed absorbance is lower than the true absorbance and that the error is a function of the bandwidth ratio, as shown in figure 4. Strictly speaking, the error magnitude is 


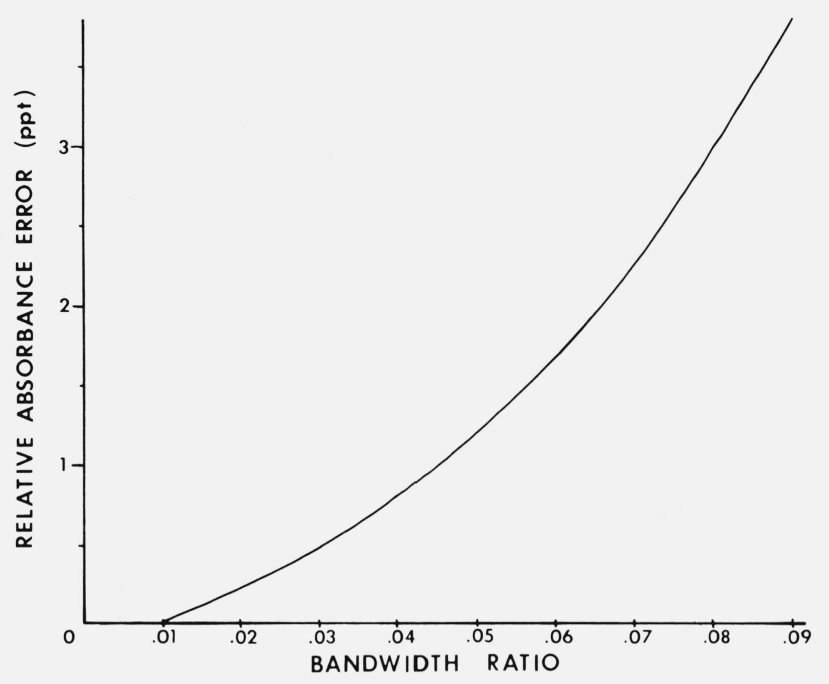

FigUre 4. Relative absorbance error (parts per thousand) due to finite slit width $(9,10)$.

also a function of absorbance, but this dependence is negligible as long as the bandwidth ratio is less than 0.4 [9].

\section{F. Stray Radiation}

As used here, stray radiation means radiation appearing at the detector with wavelengths outside the envelope defined by the slit-width and monochromator dispersion. This radiation may be either unabsorbed or partially absorbed by the sample. The variation with wavelength of source intensity, monochromator transmission and detector sensitivity are all factors which affect the amount of stray radiation present. For the case where the radiation is unabsorbed it can be shown [11] that the observed absorbance is less than the true absorbance by an amount, $\Delta A$, equal to

$$
\Delta A=\log \left(1-r+10^{A} r\right)
$$

where $r$ is the fraction of stray radiation present. Table 1 shows the relative absorbance error caused by the presence of 0.1 percent and 0.01 percent unabsorbed stray radiation. It must be emphasized, however, that if a solution has intense absorption bands at other than the wavelength for which the monochromator is set, the mathematical formulation is considerably more complex. The error may be somewhat less than is shown in table 1 , although there is also the case where the fraction of stray radiation absorbed is greater than the fraction absorbed at the wavelength of interest. In this case, the observed absorbance is greater than the true absorbance. Measurement of stray light has been discussed by Slavin [12] and Poulson [13] among others and extensive correction tables given by Opler [14]. Naturally, the fraction of stray light appearing at the detector will be relatively high in those regions where source output or detector sensitivity is low, or when working at high absorbances.
TABLE 1. Absorbance error due to unabsorbed stray radiation

\begin{tabular}{rrr}
$A$ & $\frac{\Delta A}{A} \times 1000$ (parts per thousand) \\
\cline { 2 - 3 } 0.1 & 1.12 & $r=0.0001$ \\
.2 & 1.27 & 0.11 \\
.3 & 1.44 & .13 \\
.4 & 1.64 & .14 \\
.5 & 1.88 & .16 \\
.6 & 2.15 & .19 \\
.7 & 2.48 & .22 \\
.8 & 2.87 & .25 \\
.9 & 3.34 & .29 \\
1.0 & 3.89 & .33
\end{tabular}

\section{G. Wavelength and Absorbance Accuracy}

The spectrophotometer should be calibrated for both wavelength and absorbance as close as possible to the points where they will be used. Obviously the measurements will be no better than the uncertainty of the calibration system. The error in absorbance at an absorbance maximum, due to a systematic bias in wavelength setting, is a function of the width of the absorption band of the compound of interest. The relative absorbance error is shown in figure 5, and was calculated assuming a Gaussian band shape and

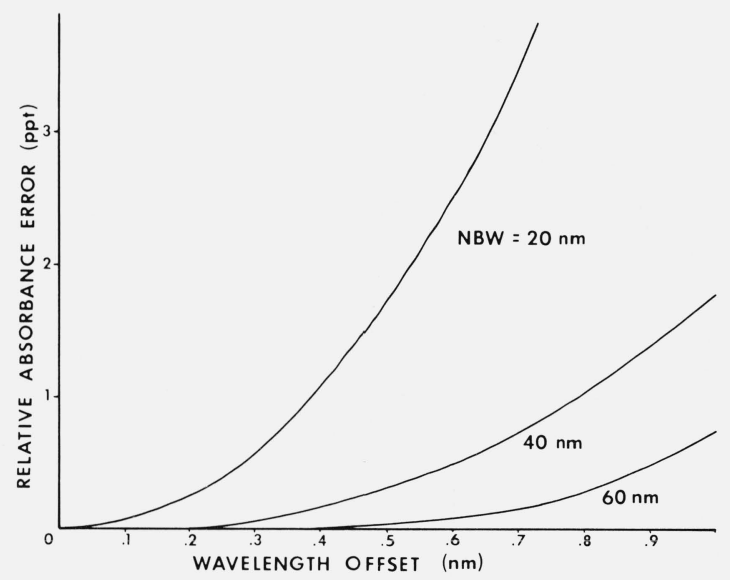

FigURE 5. Relative absorbance error (parts per thousand) due to wavelength scale inaccuracy, for absorption bands of various natural bandwidths (NBW).

neglecting the effect of finite slit-width. With regard to absorbance calibration, it may be noted that if it is feasible to use solutions as absorbance standards, the systematic errors due to path-length, beam alignment and multiple reflections may be partially compensated in the calibration. On the other hand, if the accuracy of the photometric readings is established by other, more sensitive means such as testing the summation of photon flux (light addition principle), then these 
errors must be accounted for separately.

The important sources of systematic error in spectrophotometry have been summarized in table 2 . As used in the table, a positive error is one for which the apparent absorbance is greater than the true absorbance.

TABLE 2. Systematic error sources in spectrophotometry

$\Delta A / A$ Range

(parts per thousand)

Solution temperature

Solute apparent specific volume

Buoyancy correction

Cell path-length

Cell orientation

Multiple reflections

Finite slit-width

Stray radiation

Wavelength offset obtained near $A=1.0$ and does not vary significantly between $A=0.5$ and $A=1.5$.

It should be kept in mind that the random error is actually a complex function of several variables, and the consensus is that the procedure to be recommended is to experimentally determine $\Delta A \mid A$ as a function of absorbance for the particular instrument to be used. After appropriate steps have been taken to minimize the error associated with each measurement, the residual random error component may be reduced further by performing multiple measurements. This will be commented on in the following section.

\section{Propagation of Error}

After all errors discussed above have been minimized and measured, it is necessary to ask how the residual error components combine to give an overall uncertainty in the molar absorptivity. The simplest case is that where only single measurements are made, in which case the errors in weighing and in reading solution absorbance will be propagated as systematic errors. If $F$ is any function of three independent variables, $F=f(a, b, c)$, with associated bounds of systematic error, $\Delta a, \Delta b$, and $\Delta c$, the bound for the error in $F$ is given [17] by

$$
\Delta F=\left|\frac{\partial F}{\partial a}\right| \Delta a+\left|\frac{\partial F}{\partial b}\right| \Delta b+\left|\frac{\partial F}{\partial c}\right| \Delta c
$$

Applying this relation to eq (1) yields the expression for the uncertainty in $\epsilon$,

$$
\Delta \epsilon=\left(\frac{M}{b c}\right) \Delta A+\left(\frac{A M}{b^{2} c}\right) \Delta b+\left(\frac{A M}{b c^{2}}\right) \Delta c
$$

Because of the particular form of eq (1), a simplification obtains from writing the result as a relative uncertainty

$$
\frac{\Delta \epsilon}{\epsilon}=\frac{\Delta A}{A}+\frac{\Delta b}{b}+\frac{\Delta c}{c} .
$$

This equation expresses the important result that the relative error in $\epsilon$ is just the sum of the relative errors in absorbance, path length and concentration.

Finally, it is noted that the random error component of $\Delta \epsilon$ can be reduced by performing repetitive measurements. If multiple absorbance measurements are made on the same solution, $\Delta A$ may be decreased, but $\Delta b$ and $\Delta c$ are unaffected and contribute to $\Delta \epsilon$ as before. If many solutions are prepared and measured independently, both $\Delta A$ and $\Delta c$ may be reduced, although since the reduction of the random error is proportional to $\sqrt{n}$, an improvement by a factor of two or three is all that may be practical. Moreover, it is clear that systematic errors are unaffected by repetitive measurements and will always contribute a constant amount to the final uncertainty in molar absorptivity. 


\section{Spectrophotometric Accuracy in Clinical Chemistry}

Although much attention has been focused on precision in clinical analysis, there is increasing recognition of the importance of accuracy. In a thoughtful discussion on the status of the clinical laboratory sciences, Young, et al. state [18]:

"While day-to-day . . reproducibility . . . is necessary for results to be meaningful within a single institution, it is essential that the results are also accurate if they are to have long-term validity or are to be compared with results from other laboratories."

A prerequisite for accuracy in many clinical analyses is an accurately known molar absorptivity. An important example is found in clinical enzymology where measurements of enzyme activities in blood serum account for 15 to 20 percent of all tests performed in the clinical chemistry laboratory. These activities are measured spectrophotometrically as the rate of utilization of substrate or rate of appearance of product of the enzyme-catalyzed reaction, and reported values are given in units of micromoles per minute per unit volume of serum. In these tests, standardization in the usual sense is not feasible and accuracy of results is directly dependent on the accuracy of absorbance measurements and accuracy of the value assumed for the molar absorptivity of substrate or product.

The problem is complicated further by the fact that a molar absorptivity is not a solute property, but may vary markedly with changes in solvent, $\mathrm{pH}$ or concentrations of other components in the solution. This is pertinent because of the many different conditions of temperature, $\mathrm{pH}$ and buffer composition which are routinely employed in clinical enzymology. A panel appointed by the International Federation of Clinical Chemists is currently engaged in considering referee methods for enzyme assays in clinical chemistry. It is significant that the goal of the panel is to recommend methods with a systematic bias no greater than \pm 1 percent. If this is to be achieved, it will probably be necessary to know the molar absorptivity of the substrate or product to within one or two parts per thousand.

A compound of particular interest in this connection is nicotinamide adenine dinucleotide (NAD) which is a required coenzyme in many reactions of clinical importance. $\mathrm{NADH}$, the reduced form of NAD, has a characteristic absorbance maximum at $340 \mathrm{~nm}$, and rate reactions involving this couple are commonly followed by measuring absorbance at $340 \mathrm{~nm}$ as a function of time. The accepted value of 6220 $1 \mathrm{~mol}^{-1} \cdot \mathrm{cm}^{-1}$ for the molar absorptivity of NADH at $340 \mathrm{~nm}$ was determined in 1948 by Horecker and Kornberg [19]. It is not widely appreciated that their seven experimental values ranged from 5930 to 6310 . These authors considered 6220 to be a minimum value and estimated a possible error of 2 percent in their assigned value. Sources of error connected with the spectrophotometer were not discussed. Thus, our best estimate of this important constant may be more realistically stated as $6200 \pm 200$, and this uncertainty alone may account for as much as a 2 to 3 percent systematic bias in many of our enzyme assays.

Another area where accurate molar absorptivities could be used to advantage is that of monitoring quality of incoming reagents, especially those used as standards. Bilirubin is an example of a compound which is widely used as a standard, but which has been notorious for variations in purity among the several commercial sources. The availability of purified bilirubin from the National Bureau of Standards (SRM 916) and the determination of its molar absorptivity makes possible the elimination of an important source of systematic error in this test and makes interlaboratory comparison of results feasible. It is emphasized that monitoring incoming reagent quality is only one aspect of an effective quality assurance program in clinical chemistry. Other items which should be routinely checked include wavelength and absorbance accuracy of spectrophotometers, following the practical suggestions of Rand [20], calibration of balances and thermometers, and reagent water purity. Each of these items deserves attention in order to make further progress toward the goal of increased accuracy in clinical chemistry.

\section{Summary}

The determination of molar absorptivities with an overall uncertainty not greater than one part per thousand is possible only if consideration is given to the several sources of error which have been discussed. Systematic errors whose magnitude and direction are both known may be corrected. This leaves random errors and systematic errors for which only an estimation of magnitude is available, which are used in estimating the overall uncertainty of the molar absorptivity value from eq (10). The need for accurate molar absorptivities has been discussed, using examples in clinical chemistry. These values, used in conjunction with a comprehensive quality assurance program, form the basis for increasing accuracy in many clinical chemistry tests, which will result in clinical laboratory data which are more useful than that presently available.

\section{References}

[1] Burke, R. W., Deardorff, E. R., and Bright, D. S., in Nat. Bur. Stand. (U.S.), Tech. Note 584, O. Menis and J. I. Shultz, Eds. (1971), p. 27.

[2] Burg, W. R., and Veith, D. A., J. Chem. Educ. 47, 192 (1970).

[3] Lewis, J. E., and Woolf, L. A., J. Chem. Educ. 48, 639 (1971).

[4] Matwey, J., Analytical Chemistry Division, National Bureau of Standards, personal communication.

[5] Meehan, E. J., in Treatise on Analytical Chemistry, I. M. Kolthoff, and P. J. Elving, Eds., Part I, Vol. 5, (John Wiley \& Sons, New York, N.Y., 1964), p. 2776.

[6] Goldring, L. S., Hawes, R. C., Hare, G. H., Beckman, A. O., and Stickney, M. E., Anal. Chem. 25, 869 (1953).

[7] Hardy, A. C., and Young, F. M., J. Opt. Soc. Am. 39, 265 (1949).

[8] Eberhardt, W. H., J. Opt. Soc. Am. 40, 172 (1950). 
[9] Broderson, S., J. Opt. Soc. Am. 44, 22 (1954).

[10] Cary Instruments, Model 16 Spectrophotometer Instruction Manual.

[11] Meehan, E. J., in Treatise on Analytical Chemistry, I. M. Kolthoff and P. J. Elving, Eds., Part I, Vol. 5 (John Wiley \& Sons, New York, N.Y., 1964), p. 2776.

[12] Slavin, W., Anal. Chem. 35, 561 (1963)

[13] Poulson, R. E., Appl. Opt. 3, 99 (1964).

[14] Opler, A., J. Opt. Soc. Am. 40, 401 (1950).
[15] Ingle, J. D., and Crouch, S. R., Anal. Chem. 43, 1331 (1971).

[16] Optimum Spectrophotometer Parameters, Applied Physics Corporation Application Report AR14-2 (1964).

[17] Ku, H. H., J. Res. Nat. Bur. Stand. (U.S.), 70C (Eng. and Instr.), No. 4, 263-273 (Oct.-Dec. 1966).

[18] Young, D. S., Scott, C. D., and Cole, E. B., Clin. Chem. 17, 818 (1971).

[19] Horecker, B. L., Kornberg, A., J. Biol. Chem. 175, 385 (1948).

[20] Rand, R. N., Clin. Chem. 15, 839 (1969).

(Paper 76A5-734) 